\title{
Open repair of type la endoleak in the aortic arch: three tailored approaches
}

\author{
Sandro Gelsomino", Elham Bidar", Roberto Lorusso, Daniel M. Johnson, Jos G. Maessen, Ehsan Natour \\ Cardiothoracic Department, Maastricht University Hospital, Maastricht, The Netherlands \\ "These authors contributed equally to this work. \\ Correspondence to: Prof. Sandro Gelsomino. Department of Cardiothoracic Surgery, Cardiovascular Research Institute Maastricht-CARIM, \\ Universiteitssingel 50, 6229 ER Maastricht, The Netherlands. Email: sandro.gelsomino@maastrichtuniversity.nl.
}

Submitted Jul 13, 2018. Accepted for publication Sep 06, 2018.

doi: $10.21037 /$ jtd.2018.10.21

View this article at: http://dx.doi.org/10.21037/jtd.2018.10.21

\section{Introduction}

Endoleaks are an important complication following hybrid thoracic endovascular aortic repair (TEVAR) with an incidence ranging from $20 \%$ to $25 \%(1,2)$. There are five different types of endoleaks, which are classified based on the source of vessels that cause the inflow into the aneurysm sac (3). Type I endoleaks (EL-I) occur at either the proximal (Ia) or distal (Ib) attachment sites and can be seen during insertion of the initial stent graft or during a follow-up surveillance imaging exam. EL-I may be secondary to incomplete dilatation or inaccurate sizing of the stent graft, diseased aortic wall or aortic tortuosity with angulations, leading to higher chances of rupture (4). However, EL-I represent a technical failure of endovascular repair that should be corrected promptly. However, endovascular EL-I repair at the level of aortic arch is not always possible due to an improper landing zone in the ascending aorta making it technically challenging. In the present paper, we describe three cases of EL-Ia following TEVAR and we address different repair techniques.

Written informed consents were obtained from the patients for publication of the article and any accompanying images.

\section{Operative techniques}

\section{Case 1: open repair}

A 69-year-old patient had undergone hybrid TEVAR with complete debranching of the supraaortic vessels with a carotis-subclavian bypass. He developed a type Ia endoleak
(EL-Ia) (Figure 1A,B). There were no endovascular repair options due to the debranching anastomosis at the levels of landing zone 0 . Therefore, indication for open heart surgery was given aimed at aligning the proximal part of the endostent to close the endoleak.

After repeat sternotomy and sharply adhesion division, the distal part of the endograft in the ascending aorta was identified and, since the aorto-pulmonary window was dangerously adhered, we decided to proceed on pump through aortotomy. After cannulation in the ascending aorta and the right atrium, we proceeded to circulatory arrest with mild hypothermia. Antegrade perfusion to the brain and the upper body was achieved through the debranching prosthesis on the ascending aorta. After a hockey-stick incision, the inner wall and the proximal part of the stent were visible and it was ensured to the aortic wall with interrupted 4-0 pledgeted prolene (Ethicon, Somerville, NJ, USA) sutures (Figure 1C). The cardio circulatory arrest time was 15 minutes. The aortotomy was closed and the operation was completed as usual. On postoperative angioCT, no endoleak was visible.

\section{Case 2: frozen elephant trunk}

A 74-year-old female had a history of hybrid TEVAR for aortic arch aneurysm up to landing-zone 3. She developed a symptomatic type Ia endoleak seen during follow up which lead to expansion of the aneurysm towards the left subclavian artery (Figure 2A,B). Further debranching to the ascending aorta would have been dangerous due to porcelain aorta. Therefore, the plan was to replace the 

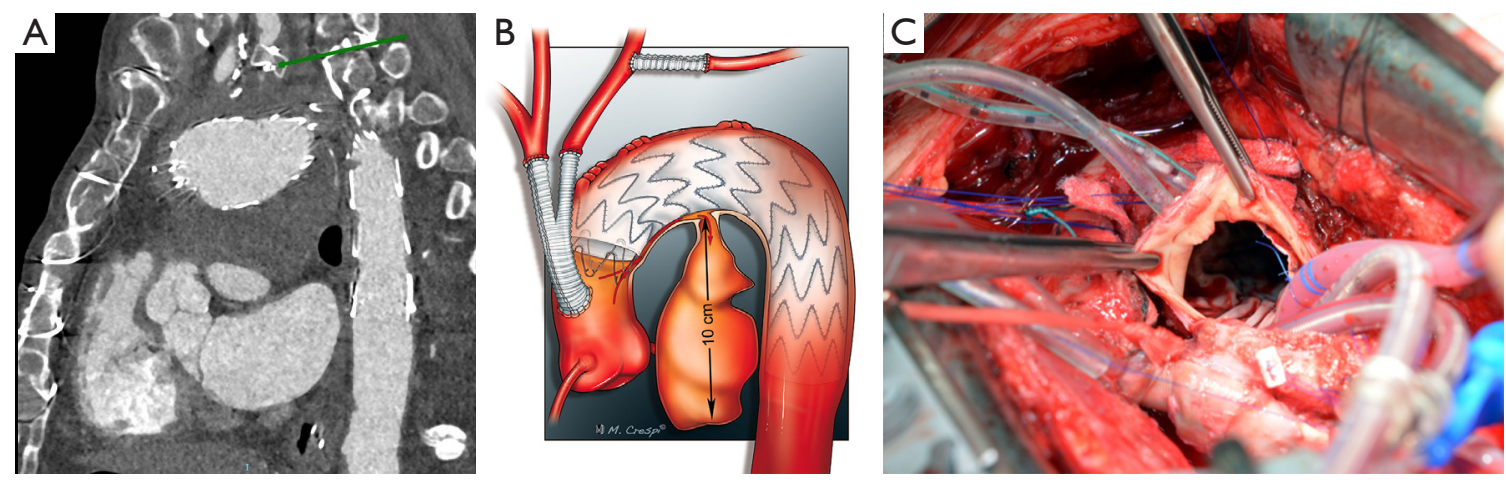

Figure 1 Case 1. (A) Preoperative CT scan; (B) the drawing shows the endoleak Ia re-feeding the aneurysm; (C) closure of the endoleak with interrupted 4-0 pledgeted prolene sutures.
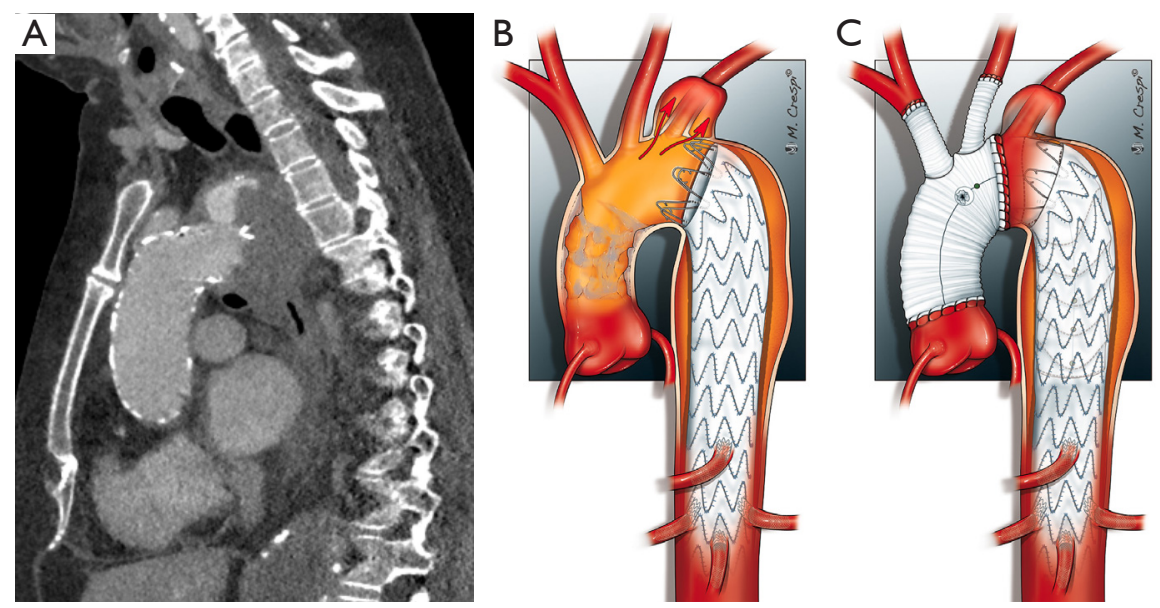

Figure 2 Case 2. (A) Preoperative CT scan; (B) type Ia endoleak seen during follow up which lead to expansion of the aneurysm towards the left subclavian artery; (C) frozen elephant trunk (see text).

ascending aorta using a frozen elephant trunk to cover the endoleak.

Under continuous electroencephalography, and aortoatrial cannulation, a moderately hypothermic circulatory arrest was established. The ascending aorta was incised and selective antegrade cerebral perfusion was initiated. On inspection, the endograft had been placed near the ostium of the subclavian artery and a partial collapse of the stent at the outer curvature was evident. A frozen elephant trunk (4-branched hybrid prosthesis, ThoraflexTM Hybrid, Vascutek Terumo, Inchinnan, UK) was inserted $15 \mathrm{~cm}$ into the endograft, and deployed accordingly. It was anastomosed at its proximal part to the aortic wall in landing zone 2, covering the subclavian artery and the entry site of the endoleak (Figure 2C). The arterial cannula was re-connected to a side arm of the Dacron prosthesis and extracorporeal circulation was re-started. Subsequently the supra-aortic vessels were re-implanted in the Dacron prosthesis and the proximal supra coronary anastomosis was performed. Post-operative angio-CT showed no endoleak.

\section{Case 3: stent in stent with complete debranching}

A 55-year-old man had previously undergone TEVAR and left-sided carotid-subclavian bypass, due to a contained rupture of the proximal descending aorta. He developed an EL-I with enlargement of the aneurysm due to malpositioning of the proximal part of the stent graft in landing zone 2 (Figure $3 A$ ). The surgical plan was to extend the prior endograft into the landing zone 0 after completing the debranching of the supra-aortic vessels.

After sternotomy and heparinization (5,000 units I.V. 


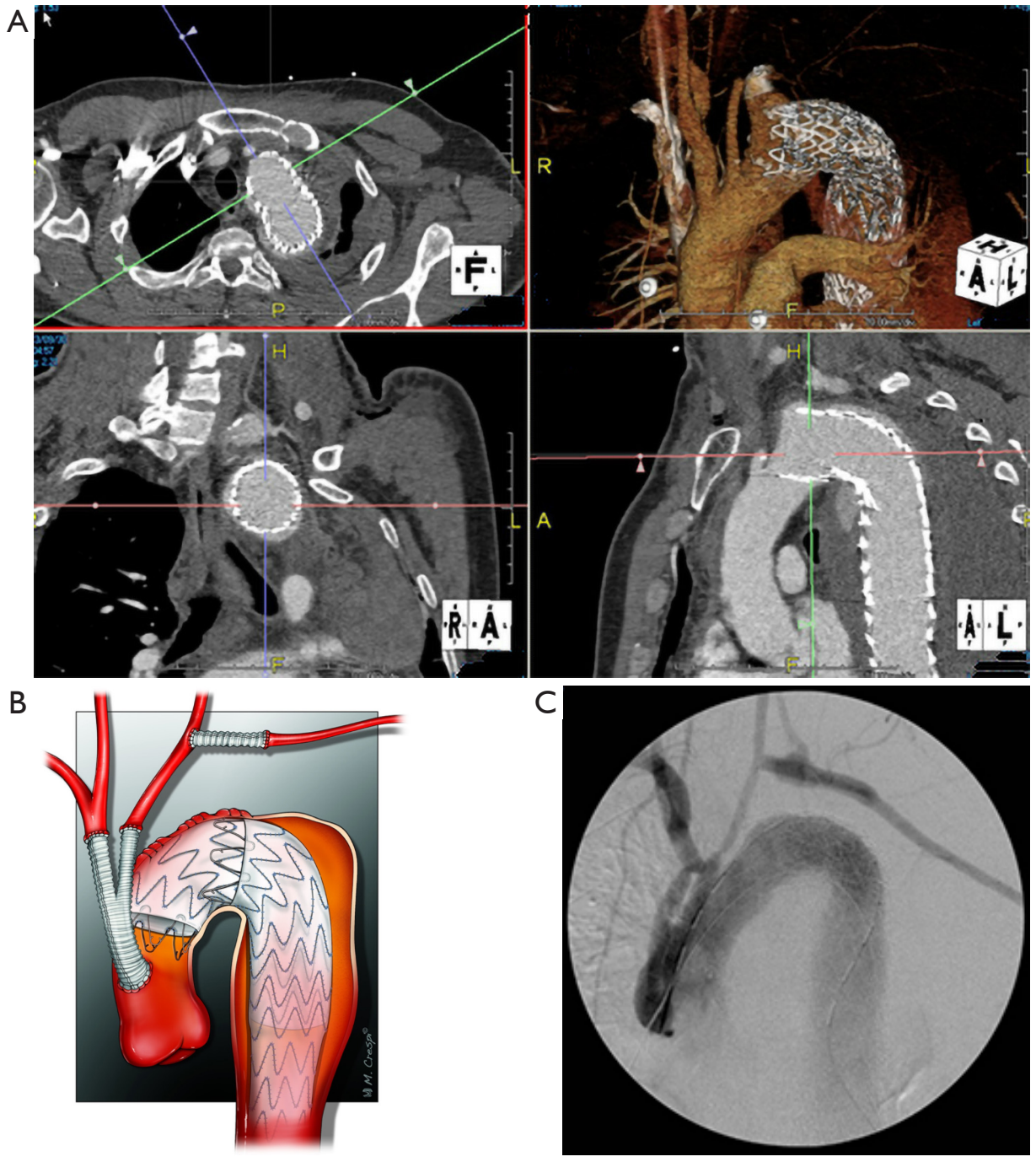

Figure 3 Case 3 (A) Type Ia at CT scan; (B) stent-in stent procedure (see text); (C) postoperative angiography.

to achieve an activated clotting time target of 200 to 300 seconds), we de-branched the supra-aortic vessels using a $16-\mathrm{mm}$ bifurcated prosthesis (Vacutek, Terumo CVS, Inchinnan, UK) which was implanted off-pump in the ascending aorta through side clamping. This allowed us to create a proper landing zone for the new endograft. The difficult aortic arch was bypassed using the throughand-through wire technique (5). This technique provides safe endostent deployment in a controlled fashion, while reducing the risk of aortic valve and coronary ostia damage. For this purpose, a $400-\mathrm{cm}, 0.035$-inch hydrophilic guidewire (Terumo Medical Corporation, Somerset, NJ, USA) was inserted through a 6-F sheath in the proximal ascending aorta and retrieved by a snare in the descending aorta which was extracted through left common femoral artery sheath. To control the proximal landing zone a stent was introduced through the ascending aorta over the through-and-through femoral-ascending aorta stiff wire and deployed to seal the endoleak with a wide overlap (Figure 3B). Post-operative angio-CT showed no endoleak (Figure 3C).

\section{Discussion}

Open repair of the aortic arch is a high-risk procedure with substantial risk of mortality and morbidity (6). Prolonged circulatory arrest, cerebra, cardiac and visceral ischemia are important concerns $(6,7)$ in addition to postoperative 
coagulation disturbances and bleeding. For these reasons, hybrid thoracic endovascular aortic repair (TEVAR) is a promising alternative $(7,8)$ with presumably lower mortality and morbidity (8-10). Nevertheless, the aortic arch is a real anatomical challenge for hybrid-TEVAR approach. Endovascular repair of aortic arch pathology is impeded due to high blood flow and local pulsatility in the arch. More importantly, the non-compliance of endostent with the inner curve of the aortic arch and the restrictions of the landing zone due to supra-aortic vessels hamper appropriate apposition of the graft, which makes this procedure prone to EL-I $(9,11,12)$. An illustrative example of this is the birdbeak configuration after TEVAR, which is an independent predictor of EL-I (13).

Complete debranching of the supra-aortic vessels to reach landing-zone 0 , seems most effective in preventing EL-I, because of a proximal seal in a non-curved ascending aorta $(9,14)$. On the other hand, because of more invasive nature of this procedure, due to sternotomy, there may be higher per-operative complications and mortality (15). Furthermore, EL-I has been described to occur in all landing zones (14).

Although most post-TEVAR complications can be managed by endovascular techniques, (10) some complications, especially EL-I, usually require open repair (16-18). Because of a large variety of anatomical variations in the diseased arch and ascending aorta, treatment strategies should be tailored to patient disease and morphology in this region.

We have described three different approaches to repair EL-I in the arch after a hybrid TEVAR. Although open surgery is an option for all patients, we tend to evaluate the least invasive measures possible.

In all cases the possibility of endovascular repair was discussed before the indication was set for open repair. In case 1 , the limitation was due to the debranching anastomosis in landing zone 0 . Therefore, an endovascular approach would have jeopardized the anastomosis to the supraaortic vessels. In case 2, only the option of an arch device would be feasible, due to a porcelain ascending aorta limiting the chances of debranching. However, a custom made arch device requires several weeks and in this case, due to symptomatic endoleak, we decided to proceed with open repair. In case 3, there was also a symptomatic endoleak which required urgent treatment. Therefore, also in this case an arch device was not feasible.

However, specific concerns arise when open repair of the endoleak is required in the aortic arch. First of all, an open anastomosis may be necessary because clamping of the aortic endograft is precluded (17). Secondly, when the supra-aortic vessels are debranched to the ascending aorta, a reoperation is much more difficult in an adhesive surgical field. This may be aggravated by systemic inflammatory reaction to the endograft (19) or local reactions to the aneurysm. Thirdly, due to mal-alignment of the stent due to arch curvature, a frozen elephant trunk may be the most reliable option because of the hand-made anastomosis at the level of endoleak. Furthermore, considering the danger of aortic valve damage with a stiff wire, a through-and through technique may be useful when landing zone 0 is pursued.

In conclusion, we believe that EL-I in the arch requires patient-tailored treatment and that, considering the possible impact of open surgery, the chance of development of EL-I should always be considered prior to hybrid-TEVAR and possible specific-treatment options be carefully evaluated.

\section{Acknowledgements}

None.

\section{Footnote}

Conflicts of Interest: The authors have no conflicts of interest to declare.

Informed Consent: Written informed consent was obtained from the patients for publication of this manuscript and any accompanying images.

\section{References}

1 Hellinger JC. Endovascular repair of thoracic and abdominal aortic aneurysms: pre- and postprocedural imaging. Tech Vasc Interv Radiol 2005;8:2-15.

2 Veith FJ, Baum RA, Ohki T, et al. Nature and significance of endoleaks and endotension: summary of opinions expressed at an international conference. J Vasc Surg 2002;35:1029-35.

3 White SB, Stavropoulos SW. Management of Endoleaks following Endovascular Aneurysm Repair. Semin Intervent Radiol 2009;26:33-8.

4 Grabenwöger M, Alfonso F, Bachet J, et al. Thoracic Endovascular Aortic Repair (TEVAR) for the treatment of aortic diseases: a position statement from the European Association for Cardio-Thoracic Surgery (EACTS) and the European Society of Cardiology 
(ESC), in collaboration with the European Association of Percutaneous Cardiovascular Interventions (EAPCI). Eur Heart J 2012;33:1558-63.

5 Brunkwall S, Ahmad W, Mylonas S, et al. The Ascending Aorta as an Exit Site for a Through-and-Through Wire in TEVAR. J Endovasc Ther 2015;22:934-7.

6 Di Eusanio M, Schepens MA, Morshuis WJ, et al. Separate grafts or en bloc anastomosis for arch vessels reimplantation to the aortic arch. Ann Thorac Surg 2004;77:2021-8.

7 Moulakakis KG, Mylonas SN, Markatis F, et al. A systematic review and meta-analysis of hybrid aortic arch replacement. Ann Cardiothorac Surg 2013;2:247-60.

8 Schoder M, Lammer J, Czerny M. Endovascular aortic arch repair: hopes and certainties. Eur J Vasc Endovasc Surg 2009;38:255-61.

9 Gelpi G, Vanelli P, Mangini A, et al. Hybrid aortic arch repair procedure: reinforcement of the aorta for a safe and durable landing zone. Eur J Vasc Endovasc Surg 2010;40:709-14.

10 Faure EM, Canaud L, Agostini C, et al. Reintervention after thoracic endovascular aortic repair of complicated aortic dissection. J Vasc Surg 2014;59:327-33.

11 Kang WC, Shin EK, Park CH, et al. Hybrid endovascular repair for aortic arch pathology: intermediate outcomes and complications: a retrospective analysis. Catheter Cardiovasc Interv 2013;82:275-82.

12 Bibiloni Lage I, Calsina Juscafresa L, Delgado Domínguez
C, et al. Hybrid Repair of Aortic Arch Aneurysms with Endografting of the Ascending Aorta. J Card Surg 2016;31:341-7.

13 Ueda T, Fleischmann D, Dake MD, et al. Incomplete endograft apposition to the aortic arch: bird-beak configuration increases risk of endoleak formation after thoracic endovascular aortic repair. Radiology 2010;255:645-52.

14 Melissano G, Civilini E, Bertoglio L, et al. Results of endografting of the aortic arch in different landing zones. Eur J Vasc Endovasc Surg 2007;33:561-6.

15 De Rango P, Cao P, Ferrer C, et al. Aortic arch debranching and thoracic endovascular repair. J Vasc Surg 2014;59:107-14.

16 Neuhauser B, Greiner A, Jaschke W, et al. Serious complications following endovascular thoracic aortic stentgraft repair for type B dissection. Eur J Cardiothorac Surg 2008;33:58-63.

17 Langer S, Mommertz G, Koeppel TA, et al. Surgical correction of failed thoracic endovascular aortic repair. J Vasc Surg 2008;47:1195-202.

18 Canaud L, Alric P, Gandet T, et al. Open surgical secondary procedures after thoracic endovascular aortic repair. Eur J Vasc Endovasc Surg 2013;46:667-74.

19 Arnaoutoglou E, Kouvelos G, Koutsoumpelis A, et al. An Update on the Inflammatory Response after Endovascular Repair for Abdominal Aortic Aneurysm. Mediators Inflamm 2015;2015:945035.
Cite this article as: Gelsomino S, Bidar E, Lorusso R, Johnson DM, Maessen JG, Natour E. Open repair of type Ia endoleak in the aortic arch: three tailored approaches. J Thorac Dis 2018;10(11):6261-6265. doi: 10.21037/jtd.2018.10.21 